\title{
UMA APLICAÇÃo INGÊNUA do MÉTOdo DE MONTE CARLO: VISUALIZAÇÃO De ORBITAIS ATÔMICOS
}

\author{
MÁRIO GOTO ${ }^{1}$ \\ VERÍSSIMO MANOEL DE AQUINO ${ }^{1}$
}

GOTO, M.; AQUINO, V.M. de. Uma aplicação ingênua do método de Monte Cario: visualização de orbitais atômicos.

Semi na: Ci. Exatas/Tecnol., Londrina, v. 13, n. 4, p. 255-261, dez. 1993.

RESUMO: Apresenta-se uma técnica relativamente simples para se obter a visualização de orbitais atômicos, exemplificando pelo átomo de hidrogênio, usando o método de simulação numérica de Monte Carlo, tendo como ferramenta um microcomputador. O programa, de fácil operação, pode ser utilizado como complemento didático para a ilustração de orbitais atômicos e outros sistemas simples. A técnica pode ser utilizada para a visualização de sistemas mais complexos.

PALAVRAS-CHAVE: Método de Monte Cario; Visualização de orbitais atômicos.

\section{1 - INTRODUÇÃO}

O método de simulação numérica, popularmente conhecido como o Método de Monte Cario (KALOS \& WHITLOCK, 1986; SÓBOL, 1976), tem uma vasta gama de aplicações para simular a evolução de sistemas muito complexos, principalmente se a evolução destes sistemas estiver associado a processos estocásticos.

$\mathrm{Na}$ Física, as aplicações do método de Monte Cario foram inicialmente desenvolvidas para tratar de problemas em mecânica estatística (BINDER \& STAUFFER, 1984; KALOS \& WHITLOCK, 1986), sendo que atualmente muitas outras áreas recorrem com sucesso ao método, notadamente em teorias na rede, como na Cromodinâmica Quântica (REBBI, 1983), por exemplo. Utilizações mais extensivas ocorrem principalmente fora da Física.
Em geral, métodos numéricos, como o é o método de Monte Cario, fornecem resultados numéricos, interpretados em tabelas e em gráficos. No entanto, nosso objetivo é diferente, pois visamos obter representações pictóricas dos sistemas estudados, que chamaremos de visualização. Escolhemos sistemas quânticos simples para. desenvolver esta técnica de visualização (partícula confinada numa caixa unidimensional e o átomo de hidrogênio) mostrando como resultado as figuras características dos orbitais atômicos. Estes sistemas tem a vantagem de terem soluções analíticas bem conhecidas. Podemos aplicar esta técnica para sistemas mais complexos, tais como sistemas atômicos e moleculares, para visualizar as estruturas das ligações químicas, por exemplo, onde observações qualitativas tornam-se importantes para a compreensão, dos sistemas. Nestes casos, no entanto, há complicações adicionais, desde que soluções analíticas

1 - Departamento de Física/CCE - Universidade Estadual de Londrina, Pr, Caixa Postal 6001, CEP 86051-970 
nem sempre são possíveis.

O ponto central da nossa técnica de simulação é a conversão da distribuição uniforme de pontos pseudoaleatórios gerados pelo microcomputador dentro do intervalo $[0,1]$ para a distribuição que queremos simular. $O$ procedimento desta conversão é a realização detestes de aceitabilidade destes pontos, no caso utilizando o teste de aceitação-rejeição de Van Neumann (KALOS \& WHITLOCK, 1986; SÓBOL, 1976).

$\mathrm{Na}$ secção 2, damos uma rápida idéia do Método de Monte Cario e do procedimento de Neumann para a seleção dos pontos para a conversão da distribuição uniforme para a distribuição desejada. A secção 3 trata do desenvolvimento da nossa técnica de visualização utilizando o método de Monte Cario, aplicado a dois sistemas simples e ilustrativos - uma partícula quântica confinada em uma caixa unidimensional e o átomo de hidrogênio nos quatro primeiros níveis de energia.

\section{2- O MÉTODO DE MONTE CARLO}

O nome é em referência a Monte Cario, principado de Mônaco, mundialmente conhecido pelos seus jogos de azar, e portanto às roletas, típicos aparelhos geradores de números aleatórios. Roletas, no entanto, são ineficientes para propósitos de simulação numérica, pois o método de Monte Cario é baseado em procedimentos estatísticos (FELLER, 1950), e para ser eficaz necessita de um grande número de números aleatórios gerados num curto espaço de tempo, motivo pelo qual o método somente se popularizou após o advento dos modernas computadores eletrônicos.

$\mathrm{Na}$ realidade, os computadores eletrônicos geram os chamados números pseudo-aleatórios, obtidos a partir de algoritmos matemáticos. No entanto, estes números pseudo-aleatórios obedecem a certos requisitos, de modo que quando gerados por um borr. algoritmo, são praticamente indistinguíveis, do ponto de vista detestes estatísticos, dos números realmente aleatórios. Em geral, as máquinas eletrônicas são programadas para gerarem os números pseudo-aleatórios dentro do intervalo [0,1], numa distribuição uniforme, de modo que torna-se necessário efetuar a conversão para a distribuição específica do sistema considerado. Para este propósito, recorremos à relação integral

$$
\gamma=\int_{a}^{\eta} p(x) d x
$$

Se $\gamma$ for uma variável aleatória uniformemente distribuido no intervalo $[0,1]$, pode-se mostrar que a variável aleatória $\eta$, definida pela relação $(2.1)$, obedece a distribuiçăo $p(x)$ definida no intervalo $[a, b]$ (SÓBOL, 1976). Na prática esta relação é interessante apenas nos casos em que podemos obter a expressão explícita de $\eta$ em função de $\gamma$, isto é, $\eta(\gamma)$ Neste caso, para obtermos a variável aleatória $\eta$ geramos $\gamma_{e}$ em seguida substituimos seu valor na expressåo para $\eta(\gamma)$. caso mais simples ocorre quando a função $p(x)$ corresponde a uma densidade de probabilidade uniformemente distribulda

$$
p(x)=\frac{1}{(a-b)},
$$

resultando, após a integração e resolução da equaçăo algébrica, na relação linear

$$
\eta=\gamma(b-a)+a .
$$

Em problemas reais, a conversão do parâmetro $\gamma$ para o parâmetro $\eta$ nảo é tão trivial, a integração nem sempre sendo possivel, ou quando possivel, a equaçäo algébrica resultante não apresenta uma solução analítica simples. Nestas situaçōes, devemos recorrer aos procedimentos numéricos de conversāo.

Para o nosso propósito, usamos o procedimento de Neumann (SÓBOL, 1976) para a conversão da distribuiçăo das variáveis aleatórias. Tomamos inicialmente um par de números aleatórios (ou pseudo-aleatórios) gerados pelo computador, e portanto pertencentes a uma distribuição uniforme dentro do intervalo $[0,1]$. Seja o par $\left(\gamma^{\prime}, \gamma^{\prime \prime}\right)$; convertemos $\gamma^{\prime}$ numa variável aleatória $\eta^{\prime}$ uniformemente distribuída no intervalo $[a, b]$ através da equação (2.3) e verificamos a desigualdade

$$
p\left(\eta^{\prime}\right)<\gamma^{\prime \prime} M_{o}
$$

para $M_{O} \circ$ máximo absoluto da função $p(x)$. Se a relação for verdadeira, aceitamos $\eta^{\prime}$, caso contrário, rejeitamos o $\operatorname{par}\left(\gamma^{\prime}, \gamma^{\prime \prime}\right)$ e realizamos um novo teste, repetindo o procedimento sucessivamente até atingir um número estatisticamente significativo das variáveis aleatórias $\eta^{\prime}$, agora na distribuição desejada.

\section{3- A TÉCNICA DE VISUALIZAÇÃO}

O objetivo da presente aplicação do método de Monte Cario é obter uma representação qualitativa de alguns sistemas físicos através da visualização de algumas das características básicas destes sistemas. Nos exemplos considerados, as distribuições de probabilidades são os principais elementos de caracterização destes sistemas. Portanto, representações qualitativas destes sistemas quânticos podem ser obtidas imprimindo pontos cuja densidade seja proporcional à densidade de probabilidade.

A técnica é muito simples: consiste na impressão na tela dos pontos obtidas durante o processo de simulação, como explicado na secção anterior. Necessitamos como suporte de um microcomputador, a programação sendo feita na linguagem BASIC, que tem a grande vantagem da simplicidade e da popularidade, embora possamos perder em eficiência quanto à velocidade de processa- 
mento, muito importante nas aplicações baseadas no método de Monte Cario, que em geral exigem um número muito grande de ciclos de processamento.

Iniciamos a aplicação pela partícula quântica confinada numa caixa unidimensional, útil para testar a técnica e a programação. Os resultados gráficos podem ser vistos no apêndice.

\section{1 - Partícula quântica confinada numa caixa}

Partícula quântica confinada numa caixa unidimensional é o exemplo mais simples e ilustrativo que achamos para explorar a nossa técnica de visualização. A densidade de probabilidade de uma partícula confinada numa caixa unidimensional de comprimento L (GASIOROWICZ, 1974) é

$$
p(x)=\frac{1}{L} \sin ^{2}\left(\frac{n \pi x}{L}\right)
$$

para números inteiros $n$. Neste caso, a relação integral (2.1) pode ser resolvida, resultando

$$
\gamma=\frac{\eta}{L}-\frac{1}{2 n \pi} \sin \left(\frac{2 n \pi \eta}{L}\right)
$$

No entanto, não podemos inverter esta equação numa relação algébrica simples relacionando as variáveis aleatórias $\eta$ e $\gamma$, recorrendo portanto ao procedimento de Neumann para a conversão da distribuição dos números aleatórios. Utilizamos este sistema simples, unidimensional, como um teste preliminar de programação e da aplicabilidade da técnica desenvolvida. Veja no apêndice algumas das figuras relacionadas a este sistema.

\section{2 - Visualizaçāo dos orbitais do átomo Hidrogê- nio}

Os auto-estados de energia do átomo de hidrogênio pode ser representada pelas auto-funçōes

$$
\Psi_{n l m}(r, \theta, \varphi)=R_{n l}(r) Y_{l m}(\theta) \Phi_{m}(\varphi),
$$

onde o número inteiro $n(n \geqslant 1)$ representa o número quântico principal, $I(0 \leqslant l \leqslant n)$ o número quântico orbital e*m $(|m| \leqslant l)$ o número quântico azimutal (GASIOROWICZ, 1974; PAULING \& WILSON, 1935).

Trabalhando em coordenadas retangulares, a distribuição espacial da densidade de probabilidade do elétron orbital é

$$
p(x, y, z)=\left|\bar{\Psi}_{n l m}(x, y, z)\right|^{2},
$$

a nossa visualizaçäo mostrando os orbitais seccionados no plano $x z(y=0)$. As figuras dos orbitais tem simetria rotacional ao redor do eixo $z$ quando usamos os autoestados de $L_{Z}$

$$
\Phi_{m}(\varphi)=\exp (\operatorname{sim} \varphi)
$$

Os procedimentos de programação serão discutidos somente para o estado fundamental, caracterizado pelo conjunto dos números quânticos $(n, l, m)=(1,0,0)$, e pela auto-função

$$
\Gamma_{100}=\frac{1}{\pi^{1 / 2} a_{o}^{3 / 2}} \exp \left(-r / a_{o}\right),
$$

onde $a_{O}\left(=0,529 \cdot 10^{-9} \mathrm{~cm}\right)$ é o raio de Bohr. A densidade espacial associada é, adotando o raio de Bohr como unidade de comprimento,

$$
p(x, y, z)=e^{-2 . r},
$$

onde

$$
r=\left(x^{2}+y^{2}+z^{2}\right)^{1 / 2},
$$

com máximo absoluto $M_{O}=1 \mathrm{em} r=0$. Na equaçäo (3.7) desprezou-se o fator multiplicativo constante, irrelevante no método de Monte Carlo. O presente sistema é tridimensional, mas a visualização será restrita ao plano $x z$, que corresponde a $y=0$. Dentro do procedimento de Neumann, num sistema bidimensional, necessitamos sortear uma tripla de números aleatórios, $\left(\gamma^{\prime}, \gamma^{\prime \prime}, \gamma^{\prime \prime \prime}\right)$, o par $\left(\gamma^{\prime}, \gamma^{\prime \prime}\right)$ devendo ser convertido para $\left(\eta^{\prime}, \eta^{\prime \prime}\right)$ através de equaçōes lineares do tipo (2.3). A seguir, para a funçāo densidade de probabilidade definida por (3.7), testamos a relação de desigualdade

$$
p\left(\eta^{\prime}, \eta^{\prime \prime}\right)<\gamma^{\prime \prime} M_{o}
$$

se for verdadeira, aceitamos este par como coordenadas de um ponto no espaço $x z$; se for falsa, rejeitamos e reiniciamos o processo, tantas vezes quantas sejam necessárias para obter uma figura bem definida após a impressāo dos pontos na tela. São necessários da ordem de três a cinco mil pontos. A técnica foi aplicada para níveis de energia $n=1$ a $n=4$, incluindo o efeito Stark no nível $n=$ 2, que causa deformação nos orbitais. Uma coletânea de figuras auto-explicativas é apresentada no apêndice, da maneira como aparece na tela do microcomputador.

\section{4 - CONCLUSÕES}

Mostramos que o método de simulação de Monte Cario pode ser usado para propósitos de visualização de alguns sistemas físicos simples, usando microcomputador como ferramenta, exemplificados aqui por várias figuras representando os orbitais dos vários níveis do átomo de hidrogênio, em secções no plano $x z$.

Baseado em algoritmos matemáticos bem definidos, tal técnica evita possíveis distorções que possam ocorrer quando tentamos traduzir equações matemáticas em figuras qualitativamente compreensíveis, passíveis a retoques artísticos subjetivos. 
Pode-se aplicar o método em sistemas mais complexos, por exemplo, para a visualização de estruturas moleculares. Os programas disponíveis estão montados visando essencialmente aplicações didáticas.

\section{APÊNDICE}

Apresentamos neste apêndice uma seleção de figuras auto-explicativas, como é vista na tela do microcomputador.

Aplicacao didatica do uso do metodo de Monte Carlo

Simulacao de orbitais: particula confinada nuna

caixa unidimensi onal
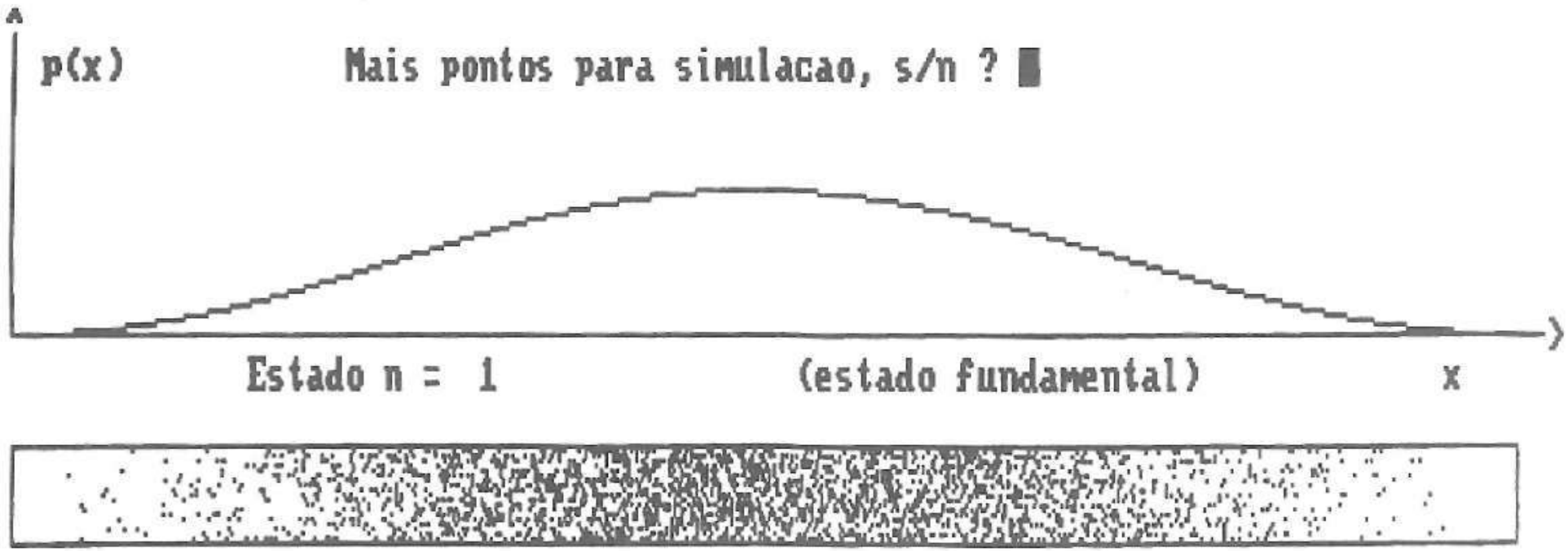

No. de ptos, lancados:

No. de ptos, para simulacao: 3900

Aplicacao didatica do uso do metodo de Monte Carlo

Simulacao de orbitais: particula confinada nuna

caixa unidimensi onal
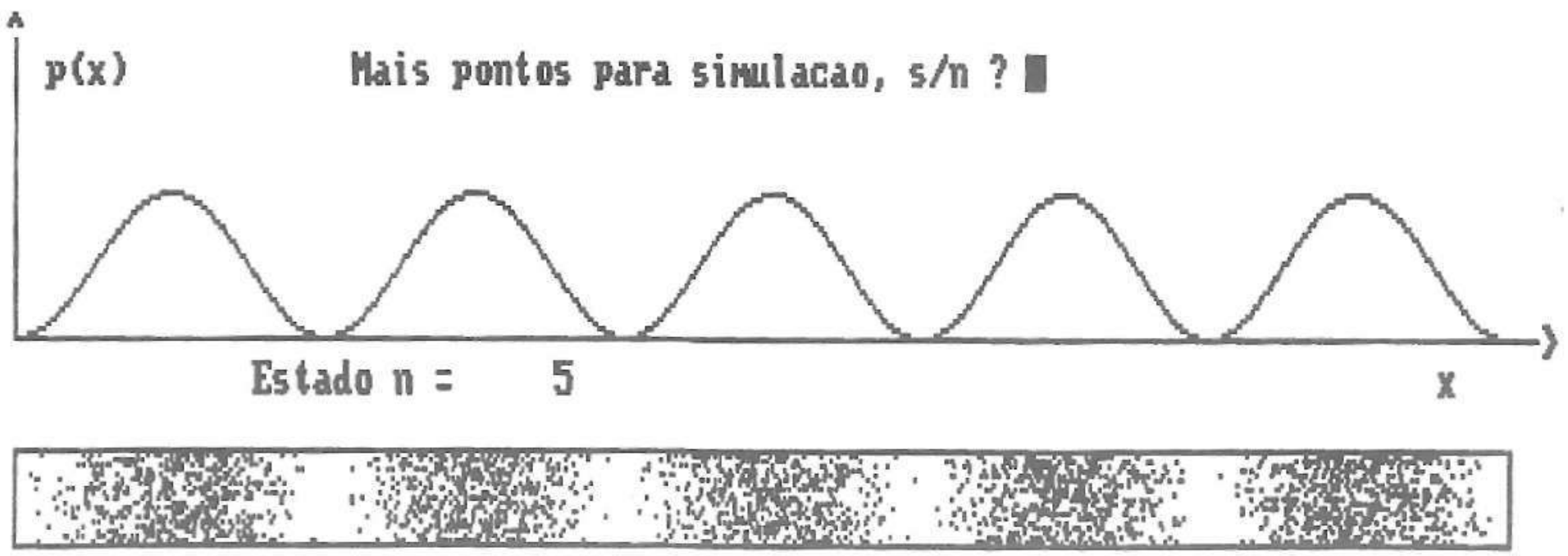

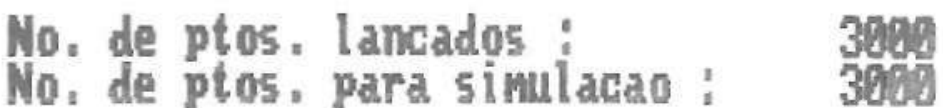




\section{Simulacao Visual de Orbitais Atomicos}

Metado de Monte Carlo:

Atomo de hidrogenio,

estado: $1,0,0$

Muda escala, $s / n$ ?

$\longmapsto=1$ raio(s) de Bohr

Ptos, lancados: 5090

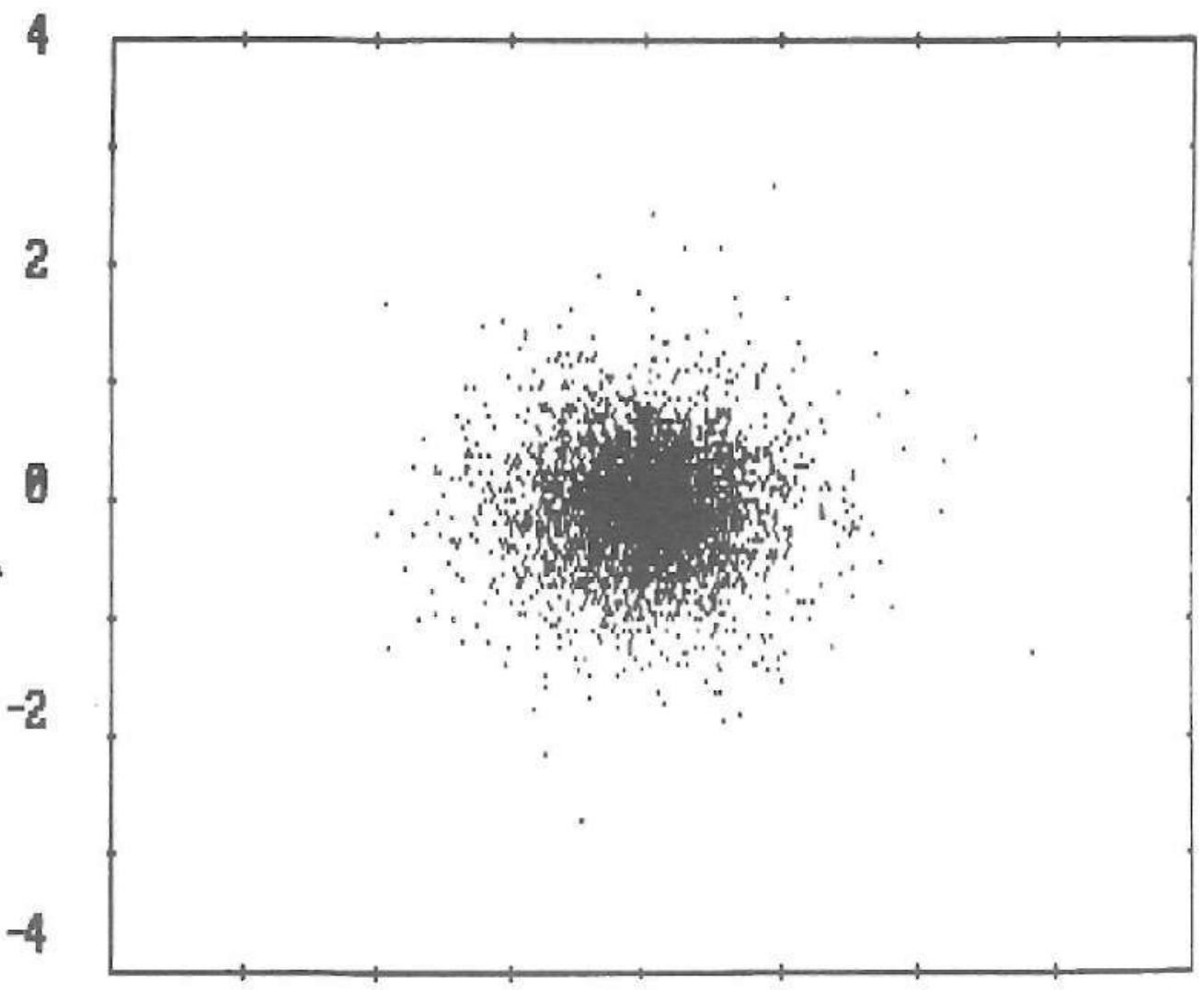

Simulacao Visual de Orbitais Atomicos

Metodo de Monte Carlo:

Atono de hidrogenio,

estado : $4,1,0$

Muda escald, $5 / \mathrm{n}$ ?

32

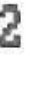




\section{Simulacao Visual de Orbitais Atomicos}

Metodo de Monte Carlo:

Atomo de hidrogenio,

estado: $4,3,0$

Huda escala, $5 / n$ ?

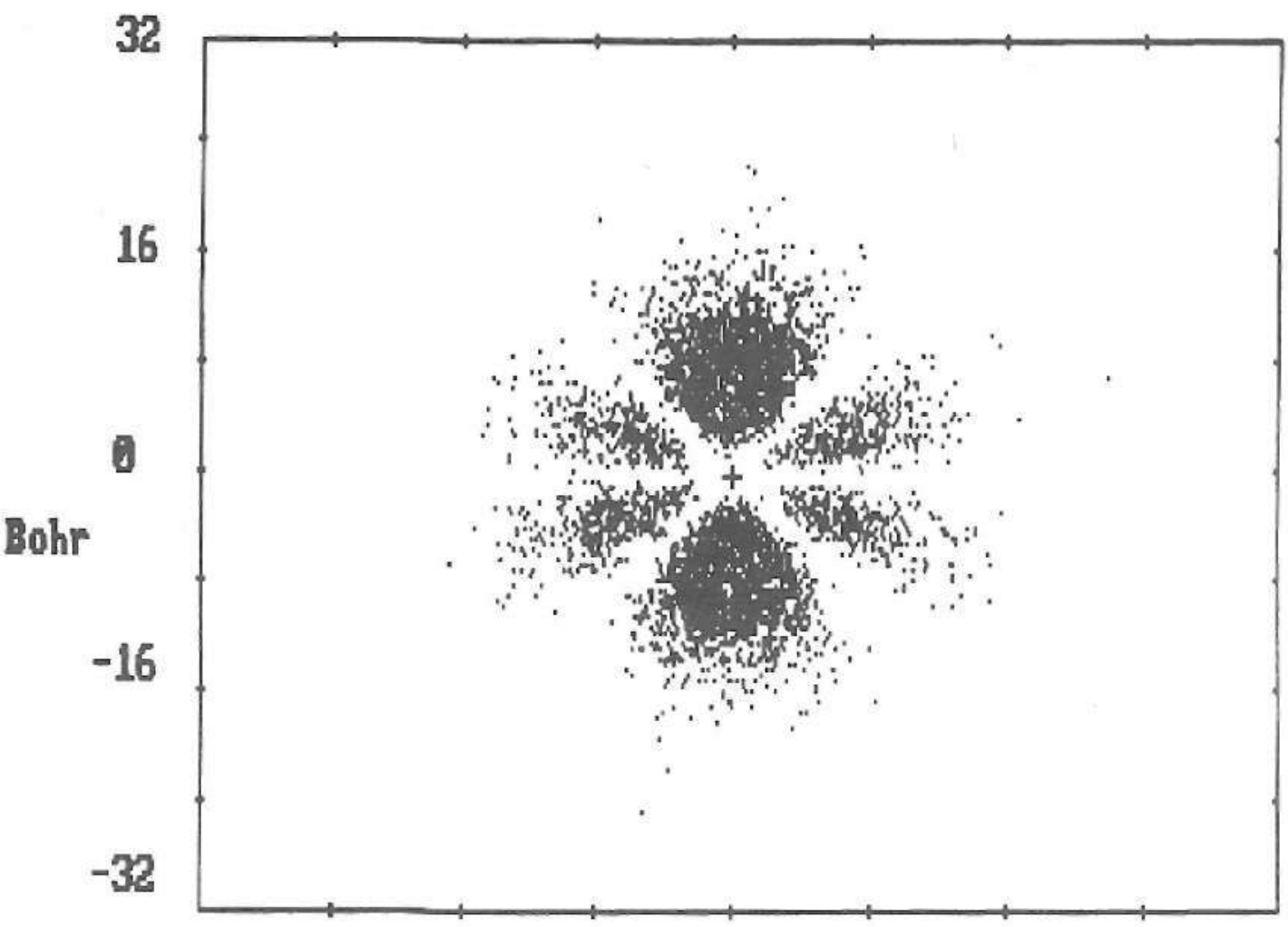

Ptos, lancados : 5990

No. de ptos. : 50 do

Simulacao Visual de Orbitais Atomicos

Metodo de Monte Carlo:

Atomo de hidrogenio,

Efei to Stark

Estado (200 - 210)

Muda escala, $s / n$ ?

$\longmapsto=2$ raio(s) de Bohr

B

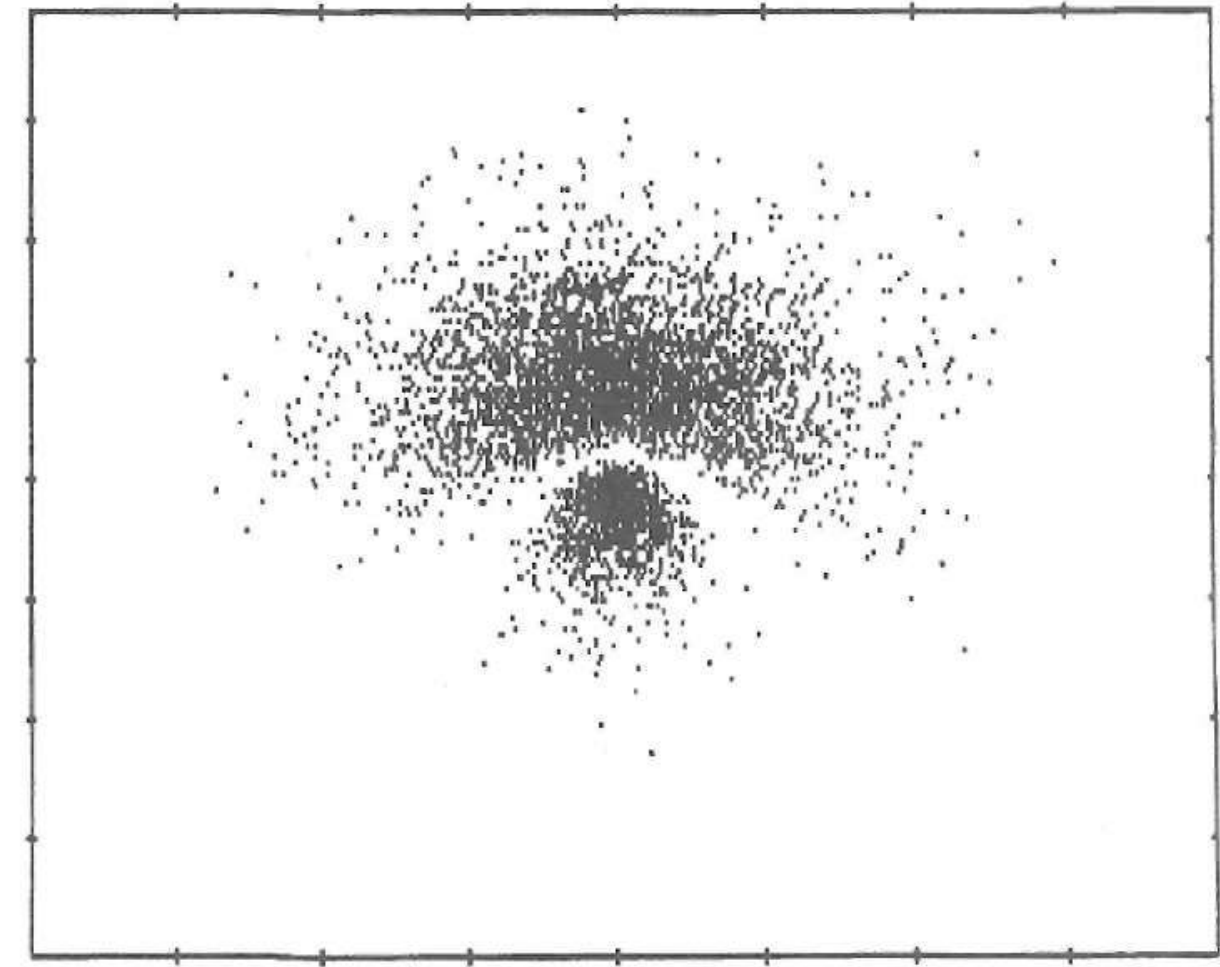

$\begin{array}{l:l}\text { Ptos lancados } & 5090 \\ \text { No. de ptos. } & 5000\end{array}$ 
GOTO, M.; AQUINO, V.M. de. A naive application of Monte Carlo simulation method: visual simulation of orbitais.

Semina: Ci. Exatas/Tecnol., Londrina, v. 13, n. 4, p. 255-261, Dez. 1993.

ABSTRACT: We present a simples technique, using the Monte Carlo Simulation Method aided by a microcomputer as a tool, to visualize atomic orbitais. The software program, applied to the hidrogen atom as an example, is easy to handle and can be used as a teaching aid to illustrate atomic orbitais as well as other simple systems. This technique can also be used for visualization of more complex systems.

KEY-WORDS: Monte Cario Simulation Method; Atomic orbitais visuaiization.

\section{REFERÊNCIAS BIBLIOGRÁFICAS}

\section{BINDER, K.; STAUFFER, D. Appllcations of the Monte Carto methods in statlstlcal physls - Topics in Current Physics, Barlin, Spinger-Vertag, v. 36, 1984.}

FELLER, W. Probability theory and its applications. New York: John Wiley \& Sons, 1950.

GASHOROWCZ, S. Quanhum Physics Naw York: John Wiley \& Sons, 1974.
KALOS, M.H.; WiHtLoCK, P.A. Monte Carlo Methods, Now York: John Whey \& Sons, 1986.

PAULING, L,; WLSON, E.B. Introduction to Quantum Mechanics. New York: McGraw-Hill, 1935.

REBBI, C. Lattice gauge theories and Monte Carto simulations, Singapore: World Scientfic, 1983.

Sóbol, l.M. Método de Monte Carto. Moscow: MiR, 1976.

Recebido para publicaçăo em 07/12/92 